ISSN electrónico: 2445-1355

DOI: https://doi.org/10.14201/fj2021613951

\title{
REVISIÓN SISTEMÁTICA DE TRATAMIENTOS EN INVESTIGACIÓN SOBRE EL CÁNCER
}

\section{Systematic Review of Cancer Research Treatments}

Raquel FRANCÉS CAMINERO ${ }^{1}$; Ramona MATEOS CAMPOS ${ }^{2}$

Departamento de Ciencias Biomédicas y del Diagnóstico. Área de Medicina Preventiva y Salud Pública. Facultad de Medicina. Universidad de Salamanca (USAL). Campus Miguel de Unamuno. C/ Alfonso X El Sabio, s/n. 37007 Salamanca ${ }^{1}$ raquelfcam@usal.es

22rmateos@usal.es, 34923294500 Ext.1809,677553806.

RESUMEN: Introducción. Dada la prevalencia y el impacto socioeconómico del cáncer, es de vital importancia la investigación en todas sus vertientes, que requiere una evaluación previa de la información científica disponible. Métodos. Se realizó una revisión sistemática de la información referente a tratamientos en investigación sobre el cáncer en Europa en los últimos diez años. Resultados. Las investigaciones incluidas pueden clasificarse y analizarse según tipo de estudio, tipo de cáncer, tipo de tratamiento, aspectos evaluados, localización geográfica e idioma de publicación. Se evaluó la calidad metodológica de los EC incluidos de acuerdo con la escala de Jadad. Conclusiones. Se analizaron siete tipos de investigaciones científicas. La mayoría fueron EC con una puntuación $\geq 3$ (sobre 5) en la escala de Jadad. Mayormente se referían a cánceres de mama y próstata y tratamientos de tipo farmacológico e inmunológico. Se estudió preferentemente seguridad y eficacia. Se llevaron a cabo sobre todo en regiones del centro, oeste y sur de Europa, y se publicaron en inglés.

Palabras clave: revisión sistemática; cáncer; tratamiento en investigación.

ABSTRACT: Introduction. Given the prevalence and socioeconomic impact of cancer, research in all its aspects is of vital importance, and it

Ediciones Universidad de Salamanca / @@ఠ $\quad$ FarmaJournal, vol. 6, núm. 1 (2021), pp. 39-51 
requires a prior evaluation of the available scientific information. Methods. A systematic review of information on investigational cancer treatments in Europe over the last ten years was carried out. Results. The investigations included can be classified and analyzed according to the type of study, type of cancer, type of treatment, aspects evaluated, geographic location and language of publication. The methodological quality of the included CTs was assessed according to the Jadad scale. Conclusions. Seven types of scientific investigations were analyzed. The majority were CT with a score $\geq 3$ (out of 5) on the Jadad scale. Most referred to breast and prostate cancers, and pharmacological and immunological treatments. Safety and efficacy were preferably studied. They were carried out mainly in regions of Central, Western and Southern Europe, and they were published in English.

Keywords: systematic review; cancer; research treatment.

\section{INTRODUCCIÓN}

«Cáncer» es un término genérico que designa un amplio grupo de enfermedades, de elevada prevalencia en la actualidad, con características definitorias comunes: multiplicación rápida de células anormales que se extienden más allá de sus límites habituales y pueden invadir partes adyacentes del cuerpo o propagarse a otros órganos. Se trata de la segunda causa de muerte en todo el mundo (9,6 millones de defunciones atribuibles en 2018). También es responsable de una significativa carga de morbilidad, en aumento de manera paralela al envejecimiento de la población y los estilos de vida poco saludables, y de gran impacto económico sobre los sistemas de salud (WHO, 2018).

La reducción de la mortalidad prematura por esta causa constituye una de las metas de los Estados miembros de las Naciones Unidas, reflejada en el Plan de acción mundial para la prevención y el control de las enfermedades no transmisibles 2013-2020 de la Organización Mundial de la Salud (OMS) (WHO, 2013) y en su Agenda para el desarrollo sostenible 2030, además de en la resolución de 2017 para el Control y la prevención del cáncer a través de un enfoque integral (WHA70.12) (WHO, 2017).

La investigación ha posibilitado el acceso a diagnóstico precoz y el desarrollo de tratamientos de calidad para aumentar las tasas de curación y supervivencia. Tal es su relevancia que la Agencia Internacional de Investigaciones sobre el Cáncer (IARC) promueve la colaboración internacional en la investigación en esta área (IARC, 2019). 
Sin embargo, en la actualidad aún tiene retos pendientes, por ejemplo: la mejora de la supervivencia a largo plazo, especialmente de los pacientes con tipos infrecuentes y poco conocidos de cáncer, y de la calidad de vida de quienes deben someterse a tratamientos agresivos. Para lograrlo, evaluar la información científica disponible es esencial.

\section{OBjetivos}

\subsection{Objetivo general}

Realizar una revisión sistemática (metaanálisis cualitativo u overview) para evaluar información referente a tratamientos en investigación sobre el cáncer en Europa en los últimos 10 años.

\subsection{Objetivos específicos}

1. Identificar los tipos de estudios sobre dichos tratamientos.

2. Valorar la calidad metodológica de los ensayos clínicos incluidos.

3. Clasificar las investigaciones según tipo de cáncer, tipo de tratamiento y aspectos evaluados.

4. Analizar la aplicación y descripción de dichas investigaciones.

5. Conocer los lugares en los que se desarrollan y el idioma de publicación.

\section{Materiales y métodos}

Para la elaboración de este trabajo se realizó una revisión sistemática (Beltrán, 2005) accediendo a la base de datos Medline a través del motor de búsqueda PubMed. La investigación final aplicando los criterios definitivos se llevó a cabo en septiembre de 2019.

Dada la amplitud del tema elegido, que trata de responder a la pregunta «¿qué se está investigando?», se filtraron los ítems resultantes por clase de tratamiento (en investigación), localización (Europa) y período temporal (últimos diez años). Los términos descriptores y operadores boleanos empleados y el número de resultados obtenidos en cada paso de la búsqueda se encuentran recogidos en la Figura 1. 


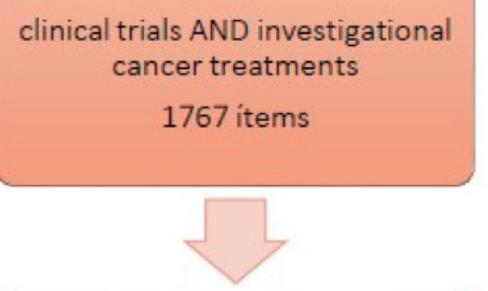

\section{clinical trials AND investigational cancer treatments AND Europe} 77 items

clinical trials AND investigational cancer treatments AND Europe

Filtro: últimos 10 años

\section{6 ítems (analizados)}

29 estudios incluidos en la revisión sistemática
17 artículos fueron excluidos por no cumplir con los criterios de inclusión:

7 por no ser posible obtener acceso completo

3 por no tener formato de artículo científico $y / o$ contener información sobre legislación (no investigaciones) - referida a investigaciones de enfermedades no oncológicas

2 por publicarse en un idioma que la autoria/tutoria de este trabajo no dominan

5 por ser articulos de opinión (no incluir un tipo de investigación válido)

Figura 1. Diagrama de flujo de la búsqueda de información.

Siguiendo la metodología de la revisión sistemática, se aplicaron unos criterios de selección específicos:

Criterios de inclusión:

- Estudio, revisión o guía-consenso de tratamientos en investigación sobre el cáncer.

- Desarrollados en países de Europa, al menos parcialmente, en los últimos diez años. 
- Investigación descrita con estructura científica completa.

Criterios de exclusión:

- Sin acceso completo.

- No comprende uno de los tipos de investigación anteriormente mencionados.

- Estructura y/o información inadecuadas.

- Idioma de publicación no dominado por la autoría de este trabajo.

Para los artículos encontrados que no eran de acceso libre se recurrió a las revistas en las que fueron publicados y a las que está suscrita la USAL.

\section{Resultados y discusión}

\subsection{Tipos de estudios}

Este apartado se subdivide según han sido clasificadas las investigaciones incluidas:

4.1.1. Ensayos clínicos

Dieciocho de las investigaciones incluidas son ensayos clínicos (EC), estudios experimentales de carácter prospectivo en los que se compara el efecto y el valor de una intervención respecto a un control en seres humanos. Son la mayoría porque se trata del descriptor empleado.

Seis (Rinnerthaler et al., Conibear et al., Kim et al., Grønhøj et al., Stein et al., Pazart et al.) son protocolos, y no incluyen los resultados del EC, pues se encuentran en fase de reclutamiento. Excluyendo estos, siete (Ravaud et al., Rödel et al., Melichar et al., Salazar et al., Scott et al., Bourgier et al., Fruehauf et al.) obtuvieron resultados favorables (indicadores en valores adecuados para pasar de fase o validar la intervención, superioridad a la referencia, balance beneficio-riesgo positivo) respecto a su objetivo, cuatro desfavorables (Valle et al., Saad et al., Fizazi et al., Sàvolt et al.) y uno de equivalencia (Von Minckwitz et al., no difiere significativamente).

Con el objetivo de personalizar la terapia, los EC se acompañan de otras investigaciones asociadas, de tipo traslacional, con distintos enfoques: identificar biomarcadores de los subgrupos de pacientes que más se beneficiarían del tratamiento (Rinnerthaler et al.) o que podrían plantear resistencias al mismo (Kim et al.), establecer factores pronóstico (Stein et al.), analizar la toxicidad en un subgrupo más concreto (Conibear et al.).

Como particularidades encontradas en este grupo: uno de los EC se refiere a pacientes en cuidados paliativos (Pazart L et al.), y a nutrición parenteral como 
intervención; otro alude al tratamiento de la xerostomía inducida por radiación (Grønhøj C et al.) (complicación del propio tratamiento oncológico).

\subsubsection{Revisiones}

Seis de las investigaciones incluidas son revisiones, estudios de carácter retrospectivo, en los que se evalúa y sintetiza información científica. Pueden ser experimentales o sistemáticas (Carceller et al., Cassier et al.), bibliográficas o narrativas (Forastiere et al., Michielin et al., Du-Thanh et al., Sternberg). Dentro del primer tipo, una es un metaanálisis cuantitativo (Carceller et al.) y otra cualitativo (Cassier et al.). Algunas están dirigidas a grupos poblacionales específicos: pediátrico (Carceller et al.), geriátrico (Du-Thanh et al.).

Casi en su totalidad obtienen resultados favorables (confirman eficacia/seguridad o mejora en la selección del tratamiento), excepto una que no difiere de la referencia (Cassier et al.).

\subsubsection{Estudios observacionales}

Una de las investigaciones incluidas es un estudio observacional (Puértolas et al.) sobre la eficacia de los factores estimulantes de colonias de granulocitos biosimilares en la prevención de neutropenia febril por quimioterapia (profilaxis de una complicación del tratamiento). Concluye con resultado favorable.

\subsubsection{Guías-consensos}

Dos de las investigaciones incluidas son guías-consensos sobre medidas de diagnóstico y tratamiento de distintos tipos de cáncer, elaborados por procedimientos distintos: la guía (Rozet et al.) sintetiza recomendaciones basándose en una revisión sistemática, mientras que el consenso (Valentini et al.) se alcanza por el método Delphi.

Ambos establecen pautas para un nuevo estándar de tratamiento a aplicar en la práctica clínica, basadas en la evidencia científica disponible, con resultado favorable.

\subsubsection{Otros}

Una de las investigaciones incluidas se refiere a un estudio de farmacoeconomía (Rule et al.). Valora la eficiencia en la administración de una nueva formulación de rituximab para un EC de fase III, concluyendo con resultado favorable. 
Otra investigación es un estudio piloto (Hughes et al.) de una aplicación tecnológica para transmitir información paciente-promotor sobre la experiencia más allá de datos clínicos. Consiste en un estudio ejecutado previamente a pequeña escala para evaluar viabilidad, tiempo y coste, y detectar errores que harían poco eficiente la investigación. El resultado fue favorable.

\subsection{Valoración de la calidad de los ensayos clínicos}

A los EC se les ha aplicado la escala de Jadad (Jadad et al., 1996) para valorar su calidad metodológica como estudios científicos según las características descritas en los artículos. El resultado aparece recogido en la Tabla 1.

TABLA 1. Evaluación de la calidad metodológica de los EC según la escala de Jadad

\begin{tabular}{|c|c|c|c|c|c|c|}
\hline Estudio & 1 & 2 & 3 & 4 & 5 & Total \\
\hline Rinnerthaler et al.* & - & - & - & - & - & - \\
\hline Von Minckwitz et al. & 1 & 1 & 1 & 1 & 1 & 5 \\
\hline Conibear et al. & 1 & 1 & 0 & 0 & 1 & 3 \\
\hline Ravaud et al.* & - & - & - & - & - & - \\
\hline Kim et al. & 0 & 0 & 0 & 0 & 1 & 1 \\
\hline Grønhøj et al. & 1 & 1 & 1 & 0 & 0 & 3 \\
\hline Stein et al. & 1 & 1 & 0 & 0 & 0 & 2 \\
\hline Rödel et al. & 1 & 1 & 0 & 0 & 1 & 3 \\
\hline Valle et al. & 1 & 1 & 1 & 1 & 1 & 5 \\
\hline Melichar et al.* & - & - & - & - & - & - \\
\hline Saad et al. & 1 & 1 & 1 & 1 & 1 & 5 \\
\hline Fizazi et al. & 1 & 1 & 1 & 1 & 1 & 5 \\
\hline Pazart et al. & 1 & 1 & 0 & 0 & 0 & 2 \\
\hline Sàvolt et al. & 1 & 0 & 0 & 0 & 0 & 1 \\
\hline Salazar et al.* & - & - & - & - & - & - \\
\hline Scott et al. & 1 & 1 & 1 & 1 & 1 & 5 \\
\hline Bourgier et al.* & - & - & - & - & - & - \\
\hline Fruehauf et al.* & - & - & - & - & - & - \\
\hline
\end{tabular}

* Se ha decidido no evaluar con esta escala los seis EC marcados, pues se trata de ensayos en fases muy tempranas en las que el diseño metodológico puede no implicar técnicas de aleatorización o enmascaramiento y ser adecuado.

Hay que tener en cuenta que, por motivos éticos, los EC con antineoplásicos se realizan con pacientes en todas las fases, no con voluntarios sanos, y no es 
adecuado utilizar como control concurrente un grupo al que se deja sin tratamiento. Por eso, y porque en ocasiones los criterios de inclusión son tan estrictos y referidos a características tan poco frecuentes que se incluyen todos los pacientes encontrados, es más habitual que se presenten EC en los que no se aleatoriza que con otros grupos de fármacos.

La mayoría de los EC evaluables obtienen una calidad metodológica de $\geq 3$ puntos sobre 5, ya que el diseño más común en fases avanzadas de los EC incluye aleatorización y enmascaramiento. Cinco de ellos alcanzan la máxima puntuación.

\subsection{Análisis de las investigaciones}

\subsubsection{Tipo de cáncer}

Los tipos de cáncer sobre los que tratan más investigaciones son el de mama y el de próstata (Tabla 2). Coinciden con los de mayor incidencia en la región de acuerdo con la OMS, aunque su tasa de mortalidad es relativamente baja. Les sigue el grupo de tumores sólidos.

TABLA 2. Clasificación de las investigaciones incluidas en la revisión sistemática según tipo de cáncer

\begin{tabular}{ll}
\hline Tipo de cáncer & Investigaciones \\
\hline Mama & Rinnerthaler et al., Von Minckwitz et al., Puértolas et al., Sàvolt \\
Próstata & Rozet., Bourgier et al. et, Saad et al., Fizazi et al., Scott et al., Sternberg CN. \\
Pulmón & Conibear et al. \\
Tumores & Ravaud et al., Carceller et al., Melichar et al., Salazar et al. \\
sólidos & Kim et al. \\
Anal & Grønhøj et al. \\
Cabeza y cuello & Forastiere et al. \\
Laringe & Stein et al., Valle et al. \\
Tracto biliar & Rödel et al., Valentini et al. \\
Rectal & Michielin et al., Du-Thanh et al. \\
Melanoma & Rule et al., Fruehauf et al. \\
Linfoma no & Cassier et al. \\
Hodgkin & Hughes et al., Pazart et al. \\
Sarcoma & Todos
\end{tabular}




\subsubsection{Tipo de tratamiento}

La mayoría de las investigaciones se refieren a tratamientos farmacológicos (quimioterapia) (Tabla 3), que son eficaces, pero con elevados niveles de toxicidad que restan calidad de vida a los pacientes. En los últimos años destacan también los tratamientos de tipo inmunológico, que permiten personalizar la terapia según las características del cáncer, dirigiendo de este modo el tratamiento al tumor y mejorando el balance beneficio/riesgo. Asimismo, las combinaciones de varios tipos de tratamientos son bastante utilizadas.

TABLA 3. Clasificación de las investigaciones incluidas en la revisión sistemática en función del tipo de tratamiento estudiado

\begin{tabular}{|c|c|c|c|c|c|}
\hline Farmacológico & Inmunológico & Radiológico & Biológico & $\begin{array}{l}\text { Combinaciones } \\
\text { de varios tipos }\end{array}$ & Otros \\
\hline $\begin{array}{l}\text { Rinnerthaler } \\
\text { et al. } \\
\text { Ravaud et al. } \\
\text { Kim et al. } \\
\text { Carceller et } \\
\text { al. } \\
\text { Stein et al. } \\
\text { Rödel et al. } \\
\text { Valle et al. } \\
\text { Melichar et } \\
\text { al. } \\
\text { Saad et al. } \\
\text { Fizazi et al. } \\
\text { Cassier et al. } \\
\text { Scott et al. } \\
\text { Fruehauf et } \\
\text { al. }\end{array}$ & $\begin{array}{l}\text { Von } \\
\text { Minckwitz } \\
\text { et al. } \\
\text { Puértolas } \\
\text { et al. } \\
\text { Michielin } \\
\text { et al. } \\
\text { Du-Thanh } \\
\text { et al. }\end{array}$ & $\begin{array}{l}\text { Conibear } \\
\text { et al. } \\
\text { Valentini } \\
\text { et al. } \\
\text { Bourgieret } \\
\text { al. }\end{array}$ & $\begin{array}{l}\text { Grønhøj } \\
\text { et al. } \\
\text { Salazar et } \\
\text { al. }\end{array}$ & $\begin{array}{l}\text { Rozet et al. } \\
\text { Forastiere } \\
\text { et al. } \\
\text { Sàvolt et al. } \\
\text { Sternberg }\end{array}$ & $\begin{array}{l}\text { Hughes A } \\
\text { et al. } \\
\text { Pazart L } \\
\text { et al. } \\
\text { Rule S et } \\
\text { al. }\end{array}$ \\
\hline
\end{tabular}

En esta revisión se han encontrado referencias a otros tipos de tratamiento y medidas complementarias: vigilancia activa (Rozet et al.), cirugía (Rozet et al., Forastiere et al., Sàvolt et al.), terapia hormonal (Rozet et al.), cambios en la metodología de administración (Rule et al.), uso de aplicaciones tecnológicas (Hughes et al.), nutrición parenteral (Pazart et al.). 


\subsubsection{Aspectos evaluados}

La mayoría de los estudios valoran más de un aspecto del tratamiento (Tabla 4). Dos investigaciones (Carceller et al., Cassier et al.) incluyen factores pronóstico.

Se evalúa preferentemente seguridad y eficacia de los tratamientos y, en menor medida, supervivencia (global o libre de enfermedad) de los pacientes tratados. Varios estudios incluyen también indicadores que valoran calidad de vida.

TABLA 4. Clasificación de las investigaciones incluidas en la revisión sistemática en función de los aspectos del tratamiento analizados.

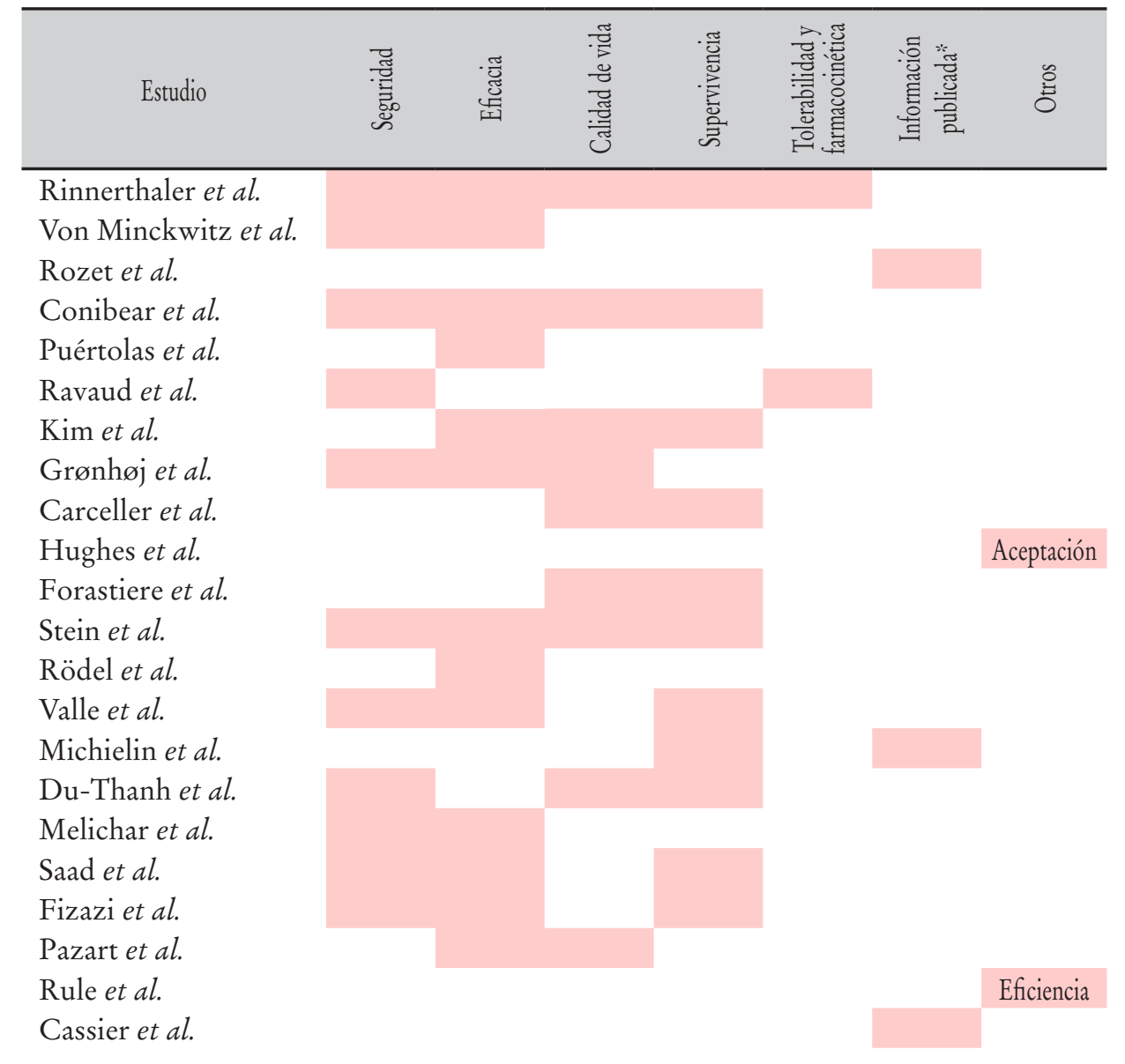


Sàvolt et al.

Salazar et al.

Scott et al.

Sternberg

Valentini et al.

Bourgier et al.

Fruehauf et al. * Se refiere a revisiones y guías-consenso, que valoran distintos aspectos de los tratamientos a partir de la
información científica disponible sobre ellos.

\subsection{Localización geográfica e idioma de publicación}

La revisión se acotó a Europa con los términos de búsqueda, aunque algunos artículos incluyen estudios desarrollados en EE. UU., Australia y Suramérica. Para las revisiones y guías-consensos se ha considerado el país en el que se han realizado, aunque los de los estudios que incluyen sean otros.

La mayoría de las investigaciones se llevaron a cabo en las zonas de mayor incidencia y mortalidad del continente, de acuerdo con el Observatorio Global del Cáncer de la IARC. Casi todas son multicéntricas, a excepción de cuatro unicéntricas (Puértolas et al., Grønhøj et al., Hughes et al., Sàvolt et al.).

Respecto al idioma de publicación, la amplia mayoría de artículos analizados está en inglés, a excepción de tres en francés (Rozet et al., Du-Thanh et al., Bourgier et al.).

\subsection{Fortalezas y limitaciones de esta revisión sistemática}

\subsubsection{Fortalezas}

- Analizar estudios con diversas particularidades, poniendo de manifiesto la amplitud y heterogeneidad de esta área de investigación. Esta variedad incluye medidas fuera del propio tratamiento oncológico: nutrición parenteral en cuidados paliativos (Pazart et al.), profilaxis (Puértolas et al.) o tratamiento (Grønhøj et al.) de reacciones adversas a las terapias agresivas, técnica de administración (Rule et al.), empleo de aplicaciones tecnológicas para comunicación paciente-promotor (Hughes et al.). También engloba grupos poblacionales específicos: pediátrico (Carceller et al.), geriátrico (Du-Thanh et al.). 
- Las revisiones sistemáticas de alta calidad de EC aleatorizados (ECAs) se consideran fuentes de información con el máximo nivel de evidencia $(1++)$ (Harbour y Miller, 2001). Dado que se han incluido también EC no aleatorizados y otros estudios científicos, se asigna un nivel $2++$, que corresponde a un grado de recomendación B.

\subsubsection{Limitaciones}

- Empleo de una única base de datos.

- Búsqueda realizada exclusivamente en inglés.

- Selección y evaluación de los estudios llevada a cabo por un solo investigador.

- Trabajar con literatura sobre tratamientos en investigación frecuentemente conlleva encontrar protocolos de EC que aún no se han realizado.

\section{Conclusiones}

1. Esta revisión sistemática ha permitido analizar, además de ensayos clínicos, otros seis tipos de investigaciones científicas. De todas ellas, diecisiete obtienen resultados favorables, cuatro desfavorables, dos de equivalencia y seis no incluyen resultados.

2. Más de la mitad de los ensayos clínicos evaluables con la escala de Jadad obtienen una calidad metodológica $\geq 3$ puntos (sobre 5). El $40 \%$ alcanza la máxima valoración.

3. Las investigaciones actuales en el ámbito oncológico se centran, principalmente, en cáncer de mama y de próstata, seguidos del grupo de tumores sólidos.

4. Los tratamientos prioritariamente estudiados son de tipo farmacológico e inmunológico y, en menor medida, combinaciones de distintos tipos.

5. La seguridad y la eficacia, así como la supervivencia de los pacientes, son los aspectos más frecuentemente evaluados.

6. La mayoría de las investigaciones se lleva a cabo en regiones del centro, oeste y sur de Europa. El idioma preferente de publicación es el inglés.

7. Los hallazgos de la investigación biomédica son la base esencial para la toma de decisiones en la práctica clínica, por lo que es de suma importancia que la información considerada sea de calidad y esté actualizada conforme a la evidencia científica, para lo cual la revisión sistemática es una herramienta de valor inestimable. 


\section{BibliografíA}

Beltrán OA. Revisiones sistemáticas de la literatura. Revista Colombiana de Gastroenterología. 2005; 20(1):60-69.

Harbour R, Miller J. A new system for grading recommendations in evidence based guidelines. BMJ. 2001; 323(7308):334-336.

International Agency for Research on Cancer (IARC) [Internet]. Lyon: IARC [actualizado 2019; citado 02 Mar 2020]. Disponible en: https://www.iarc.fr/

Jadad AR, Moore RA, Carroll D, Jenkinson C, Reynolds DJ, Gavaghan DJ et al. Assessing the quality of reports of randomized clinical trials: is blinding necessary? Control Clin Trials. 1996; 17:1-12.

World Health Organization (WHO). Global action plan for the prevention and control of noncommunicable diseases 2013-2020 [Internet]. 2013 [citado 02 mar 2020]. Disponible en: https://apps.who.int/iris/bitstream/handle/10665/94384/97892415 06236_eng.pdf

World Health Organization (WHO). Health Topics: Cancer [Internet]. Lyon: WHO [actualizado 12 sep 2018; citado 02 mar 2020]. Disponible en: https://www.who. int/news-room/fact-sheets/detail/cancer

World Health Organization (WHO). Seventieth World Health Assembly: resolutions and decisions, annexes [Internet]. Geneva: WHO; 22-31 May 2017 [citado 02 mar 2020]. Disponible en: http://apps.who.int/gb/ebwha/pdf_files/WHA70-REC1/ A70_2017_REC1-en.pdf\#page=27 
